\title{
Noether theorems and quantum anomalies
}

\author{
John Gough ${ }^{1}$, Tudor S. Ratiu ${ }^{2}$, Oleg G. Smolyanov ${ }^{3}$
}

September 17, 2018

\begin{abstract}
In this communication, we show that both infinite-dimensional versions of Noether's theorems, and the explanation of quantum anomalies can be obtained using similar formulas for the derivatives of functions whose values are measures ([1]) or pseudomeasures ([2]). In particular, we improve some results in [2].
\end{abstract}

\section{Introduction}

A quantum anomaly is the violation of the symmetry with respect to a given transformation during a quantization procedure. As such, a quantum anomaly occurs if a quantization procedure of a classical system, invariant relative to a transformation, yields a quantum system which is no longer invariant under this transformation. The calculations that are used to explain this phenomenon parallel those appearing in the proof of the infinite dimensional versions of Noether's theorems (cf. [3]). Both these calculations have two versions. In the first version, one uses integration with respect to a Feynman pseudomeasure in the case of a quantum anomaly, and alternatively integration with respect to a usual smooth $\sigma$-additive measure in the case of Noether's theorem. In the second version, both for quantum anomalies and Noether's theorem, one employs integration with respect to a Lebesgue pseudomeasure. It is worth noticing that the difference between Noether 's theorem and quantum anomalies is only that in the calculations related to the Noether theorem, one integrates the Lagrangian of the system (to get the action), whereas in the calculations related to quantum anomalies, one integrates the complex exponent of the action or of a part of the action. Using

\footnotetext{
${ }^{1}$ Institute of Mathematics, Physics and Computer Sciences, Aberystwyth University, Great Britain

${ }^{2}$ School of Mathematics, Shanghai Jiao Tong University, 800 Dongchuan Road, Minhang District, Shanghai, 200240 China and Section de Mathématiques, École Polytechnique Fédérale de Lausanne, CH-1015 Lausanne, Switzerland. Partially supported by Swiss NSF grant SwissMAP. ratiu@sjtu.edu.cn, tudor.ratiu@epfl.ch

${ }^{3}$ Faculty of Mechanics and Mathematics, Lomonosov Moscow State University, Moscow, Russia. Partially supported by the Russian Fund for Fundamental Research. smolyanov@yandex.ru
} 
these calculations, we analyze a famous contradiction between the points of view presented in the well-known monographs [4] and [5]. In particular, in [4, page 352], it is claimed that the explanation of the origin of quantum anomalies given in [5] is not correct. This criticism refers to the first, 2004 edition, of [5]. The second, 2013 edition of [5], does not address this criticism, the authors maintain their original claims, and they do not even quote [4]. Using a mathematically rigorous alternative approach, we arrive to the conclusion presented in [5]. It is worth mentioning that our approach, being infinitesimal (like in the Noether theorems), is conceptually much simpler than the global (and non-rigorous) approach presented in [4] and [5]. We also mention that in our approach to both the Noether theorem and to the quantum anomalies, we need not assume that the considered transformations are elements of any group of symmetries of the system and hence Klein's Erlangen Program has no direct relation to the subject of the present communication (already in the classical book [6], the authors do not mention any group structure on the set of transformations).

The paper is organized as follows. In section 2, we introduce some definitions of derivatives of (pseudo)measure-valued functions; most of these are more or less standard. In section 3, we present some calculations of derivatives of some functions whose values are products of a measure or pseudomeasure on a locally convex space (LCS) $E$, with a function depending on $x \in E$ and on the values at $x$ taken by another function $g$, defined on $E$, and its derivative at $x$ (the generalization to higher derivatives is straightforward) and make some remarks about how these calculations can be used in Noether-type theorems. In section 4, we get, as a corollary to calculations of the preceding section, an explanation of the origin of quantum anomalies. Like in our preceding paper [2], we consider here instead of integrals, some measures and pseudomeasures, which simplifies the situation.

We discuss here only the algebraic structure of these problems and not formulate precise analytical assumptions.

\section{Derivatives of (pseudo-)measure valued functions}

The following definitions are more or less standard. For any LCS $E$, let $\mathfrak{M}(E)$ be the vector space of all (signed) Borel $\sigma$-additive measures on $E$, equipped with the weak topology defined by a vector space $C_{b}(E)$ of some bounded functions in natural duality with $\mathfrak{M}(E)$. A function $f:(a, b) \rightarrow \mathfrak{M}(E)$ is said to be differentiable at $t \in(a, b)$ if the limit $f^{\prime}(t):=\lim _{\Delta t \rightarrow 0}(\Delta t)^{-1}(f(t+\Delta t)-f(t))$, called the derivative of $f$ at $t$, exists and if the measure $f^{\prime}(t)$ is absolutely continuous with respect to $f(t)$, denoted by $f^{\prime}(t) \ll f(t)$. In this case, the Radon-Nikodym density of $f^{\prime}(t)$ with respect to $f(t)$ is called the logarithmic derivative of $f$ at $t$ and is denoted by $\beta_{f}(t)$; thus, $f^{\prime}(t)=\beta_{f}(t) f(t)$.

Completely similar definitions can be also formulated for functions taking values 
in a space of distributions (pseudomeasures) that is defined as the space $(\mathscr{D}(E))^{\prime}$ of all continuous linear functionals on a space $\mathscr{D}(E)$ of some real or complex valued differentiable functions on $E$. We assume that $(\mathscr{D}(E))^{\prime}$ is equipped with a locally convex topology; the space $\mathscr{D}(E)$ need not coincide with the usual Schwartz space of test functions, even if $E=\mathbb{R}^{n}$. If a function $f:(a, b) \rightarrow(\mathscr{D}(E))^{\prime}$ is differentiable and if there exists a function $\beta_{f}(t)$ on $E$, which is a multiplier in $(\mathscr{D}(E))^{\prime}$ and such that $f^{\prime}(t)=\beta_{f}(t) f(t)$, then $\beta_{f}(t)$ is again called the logarithmic derivative of $f_{\nu, k}$ at $t$.

From now, $E$ always denotes an LCS.

Definition 2.1. Let $k$ be a vector field on $E$, i.e., $k$ is a Borel map of $E$ into itself, $\nu \in \mathfrak{M}(E), \varepsilon>0$. Define $S_{k}(t):=x-t k(x)$ for $t \in(-\varepsilon, \varepsilon), x \in E$, and $f_{\nu, k}:(-\varepsilon, \varepsilon) \rightarrow$ $\mathfrak{M}(E)$ by $f_{\nu, k}(t):=\left(S_{k}(t)\right)_{*} \nu$. The measure $\nu$ is called differentiable along the vector field $k$ if the function $f_{\nu, k}$ is differentiable at 0 ; then $f_{\nu, k}^{\prime}(0)$ is called the derivative of $\nu$ along $k$ and is denoted by $\nu_{k}^{\prime}$; in this case, the logarithmic derivative of $f_{\nu, k}$ at $t=0$ is called the logarithmic derivative of $\nu$ along $k$ and is denoted by $\beta_{k}^{\nu}$ (so $\beta_{k}^{\nu}$ is an almost everywhere defined function on $E$ ). If $h \in E$ and $k_{h}(x):=h$ for all $x \in E$, then the measure $\nu$ is said to be differentiable along (the vector) $h$ if it is differentiable along $k_{h}$; in this case, the logarithmic derivative of $\nu$ along $k_{h}$ is called the logarithmic derivative of $\nu$ along $h$ and is denote by $\beta^{\nu}(h, \cdot)$, i.e., $\beta^{\nu}(h, x):=\beta_{k_{h}}^{\nu}(x)$ a.e.

Definition 2.2. A vector subspace $H \subset E$ is called a locally convex (in particular, a Banach or Hilbert) subspace of $E$, if $H$ is endowed with the structure of an LCS (respectively, Banach, or Hilbert space), with respect to which the canonical embedding $H \hookrightarrow E$ is continuous.

If $H$ is a locally convex subspace of $E$ and $\nu \in \mathfrak{M}(E)$ is differentiable along each $h \in H$, then it can be shown (cf. [2]) that both mappings $H \ni h \mapsto \nu^{\prime} h \in \mathfrak{M}(E)$ and $H \ni h \mapsto \beta^{\nu}(h, \cdot)$ are linear. So $\nu$ is differentiable along each $h \in H$ if and only if the mapping $\psi_{\nu}: H \ni h \mapsto \nu_{h} \in \mathfrak{M}(E)$, where $\nu_{h}(A):=\nu(A+h)$ for any Borel set $A \subset E$, is Gâteau differentiable and for any $h, k \in H$, the measure $\left(\psi_{\nu}\right)^{\prime}(k)(h)$ is absolutely continuous with respect to $\nu_{k}$. Then the mapping $E \ni x \mapsto\left[H \ni k \mapsto \beta^{\nu}(k, x)\right] \in H^{\prime}$ is called the logarithmic derivative of $\nu$ along $H$ (if $H$ is a Hilbert space, $H^{\prime}$ is identified with $H$; in this case, the mapping $\bar{\beta}^{\nu}: E \rightarrow H$, defied by $\left(\bar{\beta}^{\nu}(x), k\right):=\beta^{\nu}(k, x)$, is called the logarithmic gradient, but we will not use this terminology).

The notion of differentiability of a measure $\nu \in \mathfrak{M}(E)$ along a locally convex subspace $H \subset E$ can be generalized in the following way. Let $H$ be a LCS, which need not be a subspace of $E$.

Definition 2.3. A map $\psi: H \rightarrow \mathfrak{M}(E)$ is called differentiable at $h \in H$, if it is Gâteau differentiable at $h$ and if, for any $k \in H$, the measure $\psi^{\prime}(h) k$ is absolutely continuous with respect to $\psi(h)$. The Radon-Nikodym derivative of $\psi^{\prime}(h) k$ with respect to $\psi(h)$ is called the logarithmic derivative of $\psi$ at $h$ along $k$ and is denoted by $\beta_{\psi}^{\nu}(h) k$. The linear mapping $k \mapsto \beta_{\psi}^{\nu}(h) k$ of $H$ into a space of functions on $E$ is called the logarithmic derivative of $\psi$ at $h \in H$. 
Remark 2.1. If $H$ is a locally convex subspace of $E, \nu$ has a logarithmic derivative along $H$, and $k$ is a vector field in $E$, such that $k(E) \subset H$, then the function $x \mapsto \beta^{\nu}(k(x), x)$ is, in general, not well defined, because for $h \in H$ the function $\beta^{\nu}(h, \cdot)$ on $E$ is defined only $\nu$-almost everywhere, the domain being dependent on $h$. A possible definition of $\beta^{\nu}(k(\cdot), \cdot)$ is given in [3], correcting an older definition of the same function presented in [1]. We recall the formula

$$
\beta_{k}^{\nu}(x)=\beta^{\nu}(k(x), x)+\operatorname{tr} k^{\prime}(x)
$$

from [1], which will be used below.

\section{$3 \quad$ Noether type theorems}

For any locally convex space $G$ and any locally convex subspace $H \subset E$, let $C_{H}(E, G)$ be the space of all mappings of $E$ into $G$ that are infinitely differentiable along $H$ (for the definition, see [2]).

Let $G, Z$ be LCS, $H$ a Hilbert subspace of $E$, and $\nu \in \mathfrak{M}(E)$. For any $g \in$ $C_{H}(E, G)$, let $\mu(g, \nu)$ be the measure on $E$ defined by $\mu(g, \nu):=L\left(\cdot, g(\cdot), g^{\prime}(\cdot)\right) \nu$, where $L \in C_{\mathscr{H}}(E \times G \times \mathscr{L}(H, G), \mathbb{C})$ and $\mathscr{H}:=H \times G \times \mathscr{L}(H, G)(L$ can be considered as a generalization of a Lagrange function).

Let $F: Z \times E \times G \times \mathscr{L}(H, G) \rightarrow E \times G$ be an infinitely differentiable mapping satisfying $F(0, x, r, \alpha)=(x, r)$ for any $\alpha \in \mathscr{L}(H, G)$. One can think that $F$ defines a family of transformations of $E \times G$ depending on a parameter $z \in Z$ and also on elements of $\mathscr{L}(H, G)$ that are, in applications, the values of the derivative of a mapping from $E$ to $G$.

Define the associated mapping $F_{Z}$ on $Z$ with values in the space of mappings from $E \times G \times \mathscr{L}(E, G)$ to $E \times G$ by $F_{Z}(z)\left(x_{1}, x_{2}, \alpha\right):=F\left(z, x_{1}, x_{2}, \alpha\right) \in E \times G$.

For a mapping $g \in C_{H}(E, G)$ and $\nu \in \mathfrak{M}(E)$, the mapping $F_{Z}$ defines the measurevalued function $F_{g, \nu}: Z \rightarrow \mathfrak{M}(E)$ in two steps. First, a $G$-valued function $g_{Z}$ is defined by its graph $\left\{F_{Z}(z)\left(x, g(x), g^{\prime}(x)\right) \mid x \in E\right\} \subset E \times G$, assuming that this set is the graph of a function. The function $g_{Z}$ can also be defined by

$$
g_{Z}\left(x_{z}\right):=F_{Z, 2}(z)\left(g\left(\left(F_{Z, 1}(z)\right)^{-1}\left(x_{z}\right)\right)\right),
$$

where, in natural notations, $F_{Z, 1}(z)(x):=\operatorname{pr}_{E} F_{Z}\left(x, g(x), g^{\prime}(x)\right)$ and $F_{Z, 2}(z)(x):=$ $\operatorname{pr}_{G} F_{Z}\left(x, g(x), g^{\prime}(x)\right)$. Second, we define a measure $\nu_{Z}:=\left(F_{Z .1}(z)\right)_{*} \nu$; then we define $F_{g, \nu}(z)$ by $F_{g, \nu}(z):=L\left(\cdot, g_{Z}(\cdot), g_{Z}^{\prime}(\cdot)\right) \nu_{Z}\left(\right.$ so, $g_{0}=g, \nu_{0}=\nu$, and $F_{g, \nu}(0)=$ $\left.L\left(\cdot, g(\cdot), g^{\prime}(\cdot)\right) \nu\right)$.

For $x \in E, \Delta \in Z$, let

$$
\begin{aligned}
& h_{1, \Delta}(x):=\left(\left(F_{Z, 1}\right)^{\prime}(0) \Delta\right)\left(x, g(x), g^{\prime}(x)\right), \\
& h_{2, \Delta}(x):=\left(\left(F_{Z, 2}\right)^{\prime}(0) \Delta\right)\left(x, g(x), g^{\prime}(x)\right),
\end{aligned}
$$




$$
h_{3, \Delta}(x):=h_{2, \Delta}^{\prime}(x) .
$$

Theorem 3.1. The logarithmic derivative $\beta_{F_{g, \nu}}$ of $F_{g, \nu}$ at 0 along $\Delta \in Z$ is given by

$$
\begin{aligned}
\left(\beta_{F_{g, \nu}}(0) \Delta\right)(x)= & L_{1}^{\prime}\left(x, g(x), g^{\prime}(x)\right) h_{1, \Delta}(x)+L_{2}^{\prime}\left(x, g(x), g^{\prime}(x)\right) h_{2, \Delta}(x) \\
& +L_{3}^{\prime}\left(x, g(x), g^{\prime}(x)\right) h_{2, \Delta}^{\prime}(x)+L\left(x, g(x), g^{\prime}(x)\right) \operatorname{tr} h_{3, \Delta}(x) \\
& +L\left(x, g(x), g^{\prime}(x)\right) \beta^{\nu}\left(h_{2, \Delta}(x), x\right) .
\end{aligned}
$$

The theorem follows from the Leibniz rule for differentiation of the product of a function and a measure, the chain rule, and formula (2.1).

Remark 3.1. The completely similar calculation can be applied when $\nu$ is not a measure, but a pseudomeasure; hence an analog of Theorem 3.1 is also available for pseudomeasures.

Remark 3.2. If $\nu$ is a translation invariant measure (in this case the dimension of $E$ has to be finite) or pseudomeasure, then $\beta^{\nu}\left(h_{2, \Delta}(x), x\right)=0$ for any $x \in E$.

Theorem 3.2. ("Noether Theorem") if $F_{Z}^{\prime}(0)=0$, then for any $\Delta \in Z$, we have

$$
\begin{aligned}
& L_{1}^{\prime}\left(x, g(x), g^{\prime}(x)\right) h_{1, \Delta}(x)+L_{2}^{\prime}\left(x, g(x), g^{\prime}(x)\right) h_{2, \Delta}(x)+L_{3}^{\prime}\left(x, g(x), g^{\prime}(x)\right) h_{2, \Delta}^{\prime}(x) \\
& \quad+L \operatorname{tr} h_{3, \Delta}(x)+L \beta^{\nu}\left(h_{2, \Delta}(x), x\right)=0 .
\end{aligned}
$$

This follows from Theorem 3.1, noting that $F_{Z}^{\prime}(0)=0$ implies $\left(\beta_{F_{g, \nu}}(0) \Delta\right)(x)=0$ for all $x \in E$.

Remark 3.3. Theorem 3.2 contains the first (direct) assertions of both theorems usually called the First and Second Noether Theorems. We quote them below from the English translation in the excellent historical book [7] and keep Noether's original notations. Noether considers functions $u_{1}(x), \ldots, u_{\mu}(x)$ for $x \in \mathbb{R}^{n}$. Then she uses an invertible transformation of $x \in \mathbb{R}^{n}$, calls the resulting variable $y \in \mathbb{R}^{n}$, and the transformed functions $v_{1}(y), \ldots, v_{\mu}(y)$. She says that the transformations are part of a group, but she never uses the group structure. Then she says that "an integral $I$ is an invariant of the group if it satisfies the relation

$$
\int \cdots \int f\left(x, u, \frac{\partial u}{\partial x}, \frac{\partial^{2} u}{\partial x}, \ldots\right) d x=\int \ldots \int f\left(y, v, \frac{\partial v}{\partial y}, \frac{\partial^{2} v}{\partial y}, \ldots\right) d x
$$

integrated over an arbitrary real domain in $x$, and over the corresponding domain in $y$." The First Noether Theorem: "If the integral $I$ is invariant under a [group] $\mathfrak{G}_{\rho} 1$, then there are $\rho$ linearly independent combinations among the Lagrangian expressions which become divergences - and conversely, that implies the invariance of $I$ under a [group] $\mathfrak{G}_{\rho}$. The theorem remains valid in the limiting case of an infinite number of

\footnotetext{
${ }^{1}$ i.e., a family of invertible transformations depending on $\rho$ parameters, not necessarily a group
} 
parameters." The Second Noether Theorem: "If the integral $I$ is invariant under a [group] $\mathfrak{G}_{\infty \rho}$ depending on arbitrary functions and their derivatives up to order $\sigma$, then there are $\rho$ identities among the Lagrangian expressions and their derivatives up to order $\sigma$. Here as well the converse is valid."

Remark 3.4. The assumption above about arbitrariness of the domain of integration means that actually one needs to study the integrands (but not the integrals), which are products of a function and of a measure.

Remark 3.5. In Theorems 3.1 and 3.2, one considers the product of a function and of a measure. Instead, one could substitute the measure $\nu$ by a product of a generalized density of $\nu$ and of the (translation invariant) Lebesgue pseudomeasure. By definition, the generalized density has the same logarithmic derivative and hence, as far as the formal calculations are concerned, nothing changes in carrying out this more general case. On the other hand, the integral with respect to the Lebesgue pseudomeasure of the generalized density is defined as a limit of some finite-dimensional integrals and it is necessary to ensure that the Leibniz rule holds; but this follows from the fact that the Leibniz rule is valid for products of functions with the measure $\nu$, because the finite-dimensional integrals in both cases are the same.

\section{Quantum anomalies}

The calculations of the preceding section, where we integrated over infinite dimensional spaces, are well adapted to problems of quantum anomalies, where one needs to integrate over an infinite dimensional space of functions, the integrand being the complex exponential of the action times either the Lebesgue pseudomeasure or the Feynman pseudomeasure and a function depending on initial data. If the derivative of the action with respect to a parameter, on which the transformations of the domain of the action depend, is equal to zero, then we can apply Theorem 3.2 .

Let $\mathscr{E}$ be the phase space of a classical Hamiltonian system, $\mathscr{E}:=Q \times P, Q$ and $P$ finite dimensional vector spaces, $\mathfrak{h}: \mathscr{E} \rightarrow \mathbb{R}$ the Hamiltonian function, and $\widehat{\mathfrak{h}}$ the pseudodifferential operator on $\mathcal{L}^{2}(Q)$ whose Weyl symbol is $\mathfrak{h}$. Let $C([0, t], \mathscr{E})$ be the space of some functions defined on $[0, t]$ taking values in $\mathscr{E}$; the elements of $C([0, t], \mathscr{E})$ are pairs $\left(\xi_{Q}, \xi_{P}\right)$, where $\xi_{Q}:[0, t] \rightarrow Q, \xi_{P}:[0, t] \rightarrow P$. Let $f \in \mathcal{L}^{2}(Q)$ be the initial data for the Cauchy problem for the Schrödinger equation $\mathrm{i} \psi^{\prime}(t)=\widehat{\mathfrak{h}} \psi(t)$ for a function $\psi: \mathbb{R} \rightarrow \mathcal{L}^{2}(Q)$. Then the solution of the Cauchy problem for this equation is given by the Feynman path integral with respect to the Lebesgue pseudomeasure $\nu$, namely,

$$
\psi(t)(q)=\int_{C([0, t], \mathscr{E})} e^{\mathrm{i} \int_{0}^{t} \mathfrak{h}\left(\xi_{Q}(\tau)+q, \xi_{P}(\tau)\right) d \tau} e^{-\mathrm{i} \int_{0}^{t}\left\langle\dot{\xi}_{Q}(\tau), \xi_{P}(\tau)\right\rangle d \tau} f\left(\xi_{Q}(t)+q\right) \nu\left(d \xi_{Q} d \xi_{P}\right),
$$

where $\langle\cdot, \cdot\rangle: Q \times P \rightarrow \mathbb{R}$ denotes the duality pairing. We do not discuss here the definition of this integral (cf. [8], where such an integral is not explicitly defined). 
Instead of the integral with respect to the pseudomeasure $\nu$, one can consider the product of the function

$$
\left(\xi_{Q}, \xi_{P}\right) \longmapsto e^{-\mathrm{i} \int_{0}^{t}\left\langle\dot{\xi}_{Q}(\tau), \xi_{P}(\tau)\right\rangle d \tau}
$$

with $\nu$, which coincides with the Feynman pseudomeasure on the space $C([0, t], \mathscr{E})$. To apply the technique of the preceding section, we now make the identifications

- $E$ is the space $C([0, t], \mathscr{E})$,

- $G$ is the space $\mathbb{C}$ of complex numbers,

- $\mu(g, \nu)$ is the pseudomeasure which is the product of the function

$$
\left(\xi_{Q}, \xi_{P}\right) \longmapsto e^{\mathrm{i} \int_{0}^{t} \mathfrak{h}\left(\xi_{Q}(\tau)+q, \xi_{P}(\tau)\right) d \tau} e^{-\mathrm{i} \int_{0}^{t}\left\langle\dot{\xi}_{Q}(\tau), \xi_{P}(\tau)\right\rangle d \tau} f\left(\xi_{Q}(t)+q\right)
$$

with the Lebesgue pseudomeasure $\nu$,

- $F_{Z}$, a formally much simpler map $\Phi$ from an auxiliary space $Z$ into the transformations of $C([0, t], \mathscr{E})$.

Then we have the following consequence of Theorem 3.2 .

Corollary 4.1. If the action and initial data are invariant with respect to transformations of $\Phi(Z) \subset C([0, t], \mathscr{E})$, then the logarithmic derivative of the pseudomeasurevalued function on $Z$, which is the sum of the logarithmic derivatives of (4) and the Lebesgue pseudomeasure, is equal to the logarithmic derivative of the Lebesgue pseudomeasure and hence need not vanish. This non-vanishing derivative is just the quantum anomaly (cf. [2]).

Acknowledgment: O.G.S. thanks the School of Mathematics of the Shanghai Jiao Tong University for the excellent working conditions provided by during his visit in July 2016, when this paper was written.

\section{References}

[1] Smolyanov, O.G. and von Weizsäcker, H., Change of measures and their logarithmic derivatives under smooth transformations, C. R. Acad. Sci. Paris Sér. I Math., 321(1) (1995), 103-108.

[2] Gough, J., Ratiu, T.S., and Smolyanov, O.G., Quantum anomalies and logarithmic derivatives of Feynman pseudomeasures, Dokl. Akad. Nauk, 465(6) (2015), 651-655; English translation, Dokl. Math., 92(3) (2015), 764-768.

[3] Smolyanov, O.G. and von Weizsäcker, H., Noether theorems for infinite-dimensional variational problems, Dokl. Akad. Nauk, 361(5) (1998), 583-586; English translation, Dokl. Math., 58(1) (1998), 91-94.

[4] Cartier, P. and DeWitt-Morette, C., Functional Integration, Cambridge University Press, 2006. 
[5] Fujikawa, K. and Suzuki, H., Path Integrals and Quantum Anomalies, Oxford University Press, 2004, second printing 2013.

[6] Gelfand, I.M. and Fomin, S.V., Calculus of Variations, revised English edition translated and edited by Richard A. Silverman, Prentice-Hall, Inc., Englewood Cliffs, N.J. 1963.

[7] Kosmann-Schwarzbach, Y., The Noether theorems. Invariance and Conservation Laws in the Twentieth Century. Translated, revised and augmented from the 2006 French edition by Bertram E. Schwarzbach. Sources and Studies in the History of Mathematics and Physical Sciences. Springer, New York, 2011.

[8] Smolyanov, O.G. and Shavgulidze, E.T., Kontinual'nye Integraly (Russian) [Continual Integrals], second edition, USSR Moscow, 2015. 